\title{
Retrospective review of children with vertigo: a 3-year experience
}

\author{
Muhammet Furkan Korkmaz ${ }^{1} \oplus$, Arzu Ekici $\odot$ \\ ${ }^{1}$ Department of Pediatrics, Bursa Yüksek IhtisasTraning and Research Hospital, Bursa, Turkey \\ ${ }^{2}$ Department of Pediatric Neurology, Bursa Yüksek IhtisasTraning and Research Hospital, Bursa, Turkey
}

\begin{abstract}
Objectives: To evaluate the age, sex, accompanying symptoms, etiologic factors and laboratory findings in children with vertigo in a tertiary research hospital.

Methods: A total of 183 children (65 boys and 118 girls), aged 3-18 (median:14), who presented with complaints of vertigo between November 2016 and September 2019 in the pediatric neurology department were examined retrospectively. Systemic and neurological examination findings, laboratory findings including complete blood count and biochemical tests (fasting blood glucose, electrolytes, liver-kidney function tests), iron, iron-binding capacity, ferritin, vitamin B12 level and thyroid function test results, electroencephalography (EEG) and magnetic resonance imaging findings were examined.
\end{abstract}

Results: The frequency of vertigo complaints were found to be higher in female gender $(p=0.008)$. The frequency of admission was significantly higher in adolescents ( $>12$ years) $(67 \%)$ compared to other age groups $(p<0.001)$. The most common cause of vertigo was benign paroxysmal vertigo of childhood (BPVC) $(23 \%)$ and orthostatic hypotension (22\%) was the second. When the relationship between the etiology of vertigo and age was examined, the most common cause was BPVC under 12 years of age, where as orthostatic hypotension was significantly more frequent in adolescents $(p<0.001)$. Headache $(41 \%)$, syncope $(27 \%)$ and nausea-vomiting $(10 \%)$ were the most common accompanying symptoms with vertigo. Epileptiform disorder was detected in $7 \%$ of patients who underwent EEG. Of 171 patients who underwent neuroimaging, $85 \%$ reported as normal and $10 \%$ had non-specific findings.

Conclusions: In children presenting with a complaint of vertigo, a detailed history including the age at when the complaint began and the accompanying symptoms, physical examination, blood pressure measurement, laboratory tests, and EEG and neuroimaging (if necessary) should be performed with a multidisciplinary approach.

Keywords: vertigo, children, dizziness, differential diagnosis

ertigo is defined as a sense of spinning dizziness and is not a common complaint in childhood [1]. The prevalence of childhood vertigo was reported to be $5.7 \%$ in a meta-analysis [2].
Patients with vertigo may experience nausea, loss of balance, sensory or visual problems, and headache in addition to the sense of dizziness [3]. The frequency of some symptoms accompanying vertigo and the un- 
derlying causes may be different in children than in adults. For example, migraine-associated vertigo (MAV) is common in children, whereas, Ménière's disease is more commonly observed in adults [4]. The studies to date have reported benign paroxysmal vertigo of childhood (BPVC), MAV, and psychogenic vertigo as the most common causes of vertigo in children [2].

Despite recent advances in medicine, the diagnosis of childhood vertigo is still based on a detailed medical history and physical examination. However, the pathogenesis of vertigo in children is different than in adults. Children have a better response to therapy and heal faster. Besides, pediatricians, otorhinolaryngologists, and pediatric neurologists often face challenges in establishing the diagnosis. The inability of the affected young children in particular, to explain the characteristics of symptoms, duration of episodes, and the provoking or accompanying factors may pose an obstacle in reaching a diagnosis [5]. Furthermore, vestibular tests are not clinically or neurophysiologically safe in young patients. However, the critical factor for the misdiagnosis and a delay in the diagnosis is the awareness threshold of clinicians about the nuances in symptomatology and treatment algorithms [6].

There is a paucity of data in the literature regarding childhood vertigo, although the first case of pediatric vertigo in the modern medical literature was described in 1962. The aim of the present study is to perform a retrospective review of pediatric patients presenting with vertigo, and thus, contribute to the literature.

\section{METHODS}

The medical records of 691 patients, aged younger than 18 years who presented to the Pediatric Neurology Outpatient Clinics at Bursa Yüksek İhtisas Training and Research Hospital (a tertiary healthcare facility) with the complaint of vertigo between November 2016 and September 2019, were retrospectively reviewed after obtaining the approval of local ethics committee (2011-KAEK-25 2019/0909). Patients with an accompanying chronic condition, patients with a history of long-term drug use of any reason, patients older than 18 years, patients who could not be etiologically classified, and those with inaccessible or missing data were excluded from the study.

One hundred and eighty-three eligible patients were evaluated for age, gender, date of admission, and the symptoms accompanying vertigo. Systemic and neurological examination findings, as well as laboratory data including complete blood count, biochemical tests (fasting blood glucose, electrolytes, liver and kidney function tests), serum iron, ironbinding capacity, ferritin, vitamin B12 levels, and thyroid function tests, were examined. The results of consultations with pediatric cardiology, pediatric endocrinology, otorhinolaryngology, pediatric psychiatry, and the results of cranial CT/MRI (Computerized tomography/Magnetic resonance imaging), EEG (electroencephalography), and MR angiography were evaluated in case a need raised.

\section{Statistical Analysis}

Categorical variables were expressed as number $(\%)$, and continuous variables were expressed as mean \pm SD for normally distributed variables and as median (minimum-maximum) if the data is not normally distributed. A chi-square test or Fisher's exact test was used to compare the frequency of qualitative variables. SPSS (Statistical Package for Science Studies) 21.0 software package was used in data analysis. A p-value less than 0.05 was considered statistically significant in all statistical tests.

\section{RESULTS}

Of 183 patients aged 3-18 years (with a median age of 14 years) who were included in the study, 65 (36\%) were male, and 118 (64\%) were female (Fig. $1)$. The frequency of vertigo in the present study was higher in females $(p=0.08)$. It was observed that the rate of presentation increased during adolescence $(>12$ years) when compared to other age groups $(p<0.001)$. The rate of presentation among girls was significantly higher in the 14-18 years age group (61\%) compared with that of other age groups $(p<0.001)$.

The most common cause of vertigo was BPVC (23\%), followed by orthostatic hypotension $(22 \%)$ (Table 1). When the relationship between vertigo etiologies and age was examined, the most common cause in patients aged 12 years and younger was 


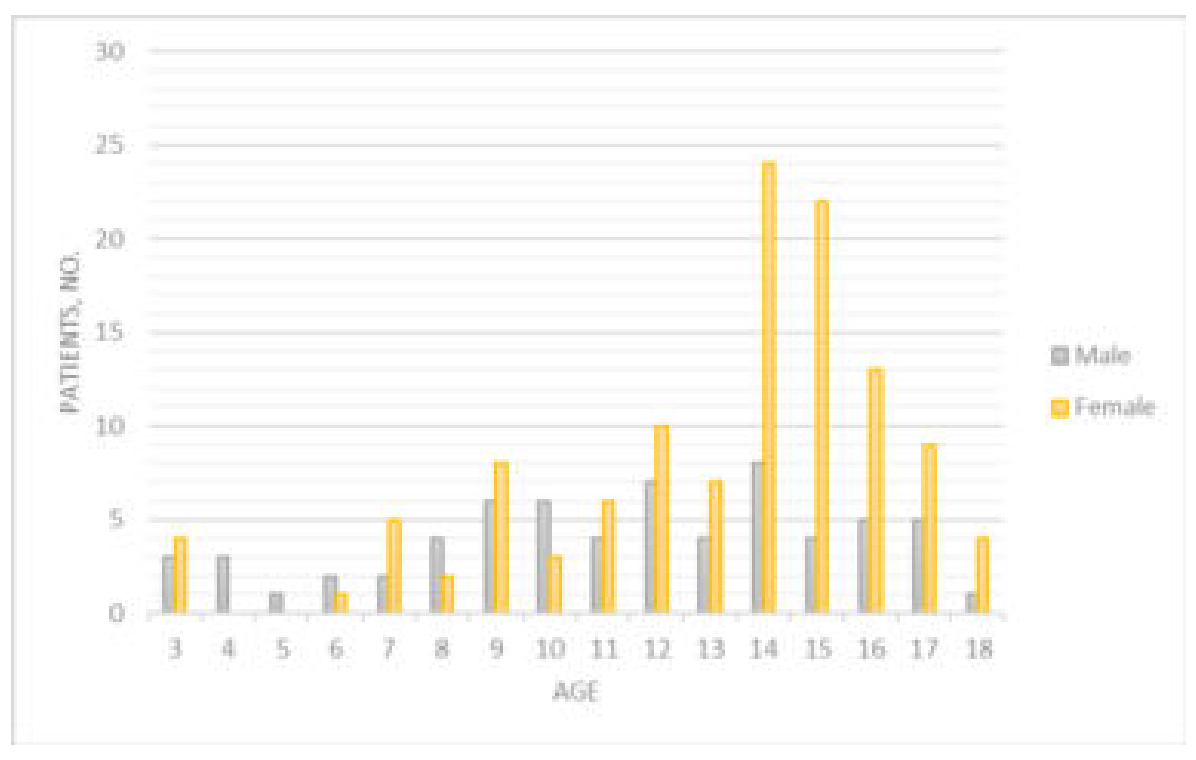

Fig. 1. Distribution of vertigo cases in terms of age and sex.

BPVC, whereas, orthostatic hypotension was significantly more common among adolescents $(p<$ 0.001) (Fig. 2). The most frequently encountered symptoms accompanying vertigo were headache $(41 \%)$, syncope $(27 \%)$, and nausea and vomiting $(10 \%)$ (Table 2).

The laboratory data revealed autoimmune thyroiditis in seven (4\%) patients, vitamin B12 deficiency in nine patients $(5 \%)$, and iron deficiency anemia in three $(2 \%)$ patients.

Of 153 patients that underwent electroencephalography, eleven $(7 \%)$ were found to have an epileptiform disorder, and these patients were diagnosed with epilepsy. Neuroimaging studies revealed normal findings in $144(85 \%)$ out of 171 patients who underwent such investigations, whereas $17(10 \%)$ patients had non-specific findings (arachnoid-pineal gland cyst, corpus callosum

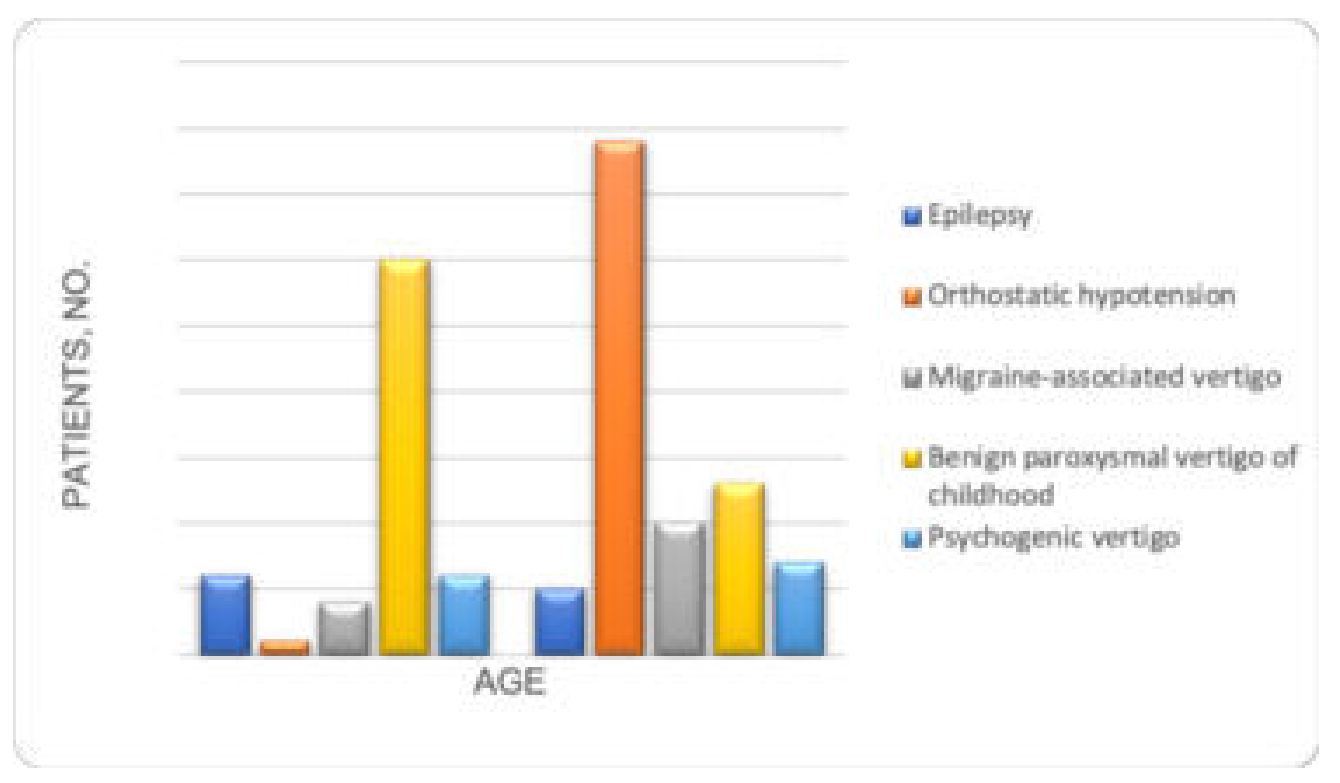

Fig. 2. The most common causes of vertigo by age group. 
Table 1. Diagnosis of 183 children with vertigo

\begin{tabular}{|c|c|c|c|}
\hline Diagnosis & n (\%) & Male/Female & Age (median [min-max]) \\
\hline $\mathrm{BPVC}$ & $43(23)$ & $18 / 25$ & $6(3-14)$ \\
\hline Orthostatic hypotension & $40(22)$ & $9 / 31$ & $15(11-18)$ \\
\hline BPPV & $15(8)$ & $6 / 9$ & $15(12-18)$ \\
\hline Migraine-associated vertigo & $14(8)$ & $5 / 9$ & $14(7-17)$ \\
\hline Psychogenic vertigo & $13(7)$ & $3 / 10$ & $11(7-16)$ \\
\hline Epilepsy & $11(6)$ & $5 / 6$ & $12(9-18)$ \\
\hline Refractive errors & $10(6)$ & $5 / 5$ & $12(3-18)$ \\
\hline Vitamin B 12 deficiency & $9(5)$ & $6 / 3$ & $15(10-17)$ \\
\hline Thyroiditis & $7(4)$ & $0 / 7$ & $15(11-17)$ \\
\hline Sinusitis & $6(3)$ & $4 / 2$ & $10(4-17)$ \\
\hline Otitis media & $5(3)$ & $2 / 3$ & $8(3-14)$ \\
\hline IDA & $3(2)$ & $0 / 3$ & $13(12-14)$ \\
\hline Mastoiditis & $2(1)$ & $0 / 2$ & $4.5(3-6)$ \\
\hline Subclavian steal syndrome & $2(1)$ & $0 / 2$ & $11.5(9-14)$ \\
\hline Posttraumatic vertigo & $1(1)$ & $1 / 0$ & 14 \\
\hline Postoperative vertigo & $1(1)$ & $0 / 1$ & 16 \\
\hline Multiple sclerosis & $1(1)$ & $1 / 0$ & 17 \\
\hline Total & $183(100)$ & $65 / 118$ & $13(3-18)$ \\
\hline
\end{tabular}

$\mathrm{BPVC}=$ Benign paroxysmal vertigo of childhood, BPPV $=$ Benign paroxysmal positional vertigo, IDA $=$ Iron deficiency anemia.

agenesis, choroid plexus papilloma, pituitary microadenoma, arachnoid granulation), four $(2 \%)$ patients had sinusitis, two (1\%) patients had mastoiditis, one patient had venous angioma and another patient had demyelinating plaques. Two (1\%) patients were reported to have a thin-caliber unilateral vertebral artery. Patients evaluated with MR angiography were diagnosed with subclavian steal syndrome.

\section{DISCUSSION}

The current study presents a retrospective review of clinical and laboratory data of pediatric patients who admitted to the pediatric neurology outpatient clinics of our tertiary healthcare center with the complaint of vertigo during a 3-year period. Vertigo is uncommon in childhood, but it represents a broad differential diagnosis comprising a wide gamut of causes [7].

Benign positional vertigo of childhood is a heterogeneous disease characterized by recurrent short episodes of vertigo attacks that occur without prior warning. It is regarded as a common cause of vertigo in children with a prevalence of $2.6 \%$ [2]. The onset of BPVC is typically before the age of four. Disease onset after eight years of age is extremely rare [8]. The 
Table 2. Symptoms accompanying vertigo

\begin{tabular}{lc}
\hline Symptoms & $\mathbf{n}(\mathbf{\%})$ \\
\hline Headache & $45(41)$ \\
Syncope & $31(27)$ \\
Nausea and vomiting & $11(10)$ \\
Convulsion & $6(6)$ \\
Tremor & $5(5)$ \\
Hypotension & $3(3)$ \\
Blurred vision & $3(3)$ \\
Numbness in hands & $2(2)$ \\
Tinnitus & $2(2)$ \\
Ataxia & $1(1)$ \\
Total & $\mathbf{1 0 9 ( 1 0 0 )}$ \\
\hline
\end{tabular}

diagnosis is established on the basis of age and typical clinical appearance after ruling out other causes of vertigo. Headache can occur in time in patients with BPVC, and the diagnosis in these patients can be changed to MAV [9]. In our study, BPVC was found to be the most common (23\%) cause of vertigo. In line with the literature, the median age of these patients was six years. In addition, BPVC was also found to be the most common cause of vertigo in the studied patients younger than 12 years. Examining more than 2,000 pediatric patients presenting with the complaint of vertigo, Wiener-Vacher [10] reported MAV (25\%) and BPVC (20\%) as the most common causes in the etiology of vertigo. Similarly, Ravid et al. [11] reported MAV (39\%), BPVC (16\%), psychogenic vertigo (13\%), and orthostatic hypotension ( $9 \%$ ) as the most frequent causes of vertigo. Consistent with the literature, the present study reports BPVC (23\%) and orthostatic hypotension (22\%) as the most prevalent causes of vertigo.

Orthostatic hypotension is frequently observed in children who undergo rapid growth (before and during adolescence), and it represents a temporary weak adaptation of the cardiovascular system to abrupt changes in body position. Vertigo often occurs in the morning after wake-up (while trying to get up rapidly) or while standing for an extended period. Vertigo can be accompanied by the feeling of dizziness, fainting, and rarely loss of consciousness. Vestibular tests prove normal. The diagnosis of orthostatic hypotension is established by demonstration of a change in arterial blood pressure when measured upon standing up quickly after having remained in the lying position for 20 minutes [10]. Orthostatic hypotension was found to be the second most common (22\%) cause of vertigo in the present study, and even higher rates were noted in the 14-16 years age group in line with the literature.

Differential diagnosis requires a detailed medical history, physical examination, and scrutinizing accompanying symptoms. Headache, tinnitus, blurred vision, and loss of balance are the most common symptoms accompanying vertigo in pediatric patients $[1,12]$. Headache $(41 \%)$, syncope $(27 \%)$, and nausea and vomiting $(10 \%)$ were the most common symptoms accompanying vertigo in the present study. Epileptic vertigo and MAV should be consired in the presence of signs accompanying vertigo such as photophobia, nausea, vomiting, smell disorders and hallucination. Migrainoid headaches are more frequent in childhood than in adults. The studies reported prevalence rates as high as $35 \%$, whereas the prevalence rate was only $6 \%$ in adults [13]. In addition to headaches, it can be seen with concomitant phonophobia, photophobia, nausea, vomiting, and smell disorders. The complaints often have an onset in childhood and are more common in girls [14]. In vertigo patients with accompanying headache, the rate of migraine-associated vertigo was $8 \%$ and more common in the girls $(64 \%)$ that was consistent with the literature. Epileptic vertigo is usually associated with signs such as complex movement hallucinations (more than simple rotational vertigo) that provoke an epileptic seizure, auditory hallucinations, symptoms of neurological localization, and loss of consciousness. These kinds of complaints absolutely require neuroimaging studies and a consultation with a pediatric neurologist [15]. Epileptiform disorders were detected in $11(7 \%)$ out of 153 patients undergoing EEG, and all these patients were diagnosed with epilepsy in our study.

Vertigo can occur secondary to infections in childhood. It can result from otitis media or middle ear effusions that are commonly seen in this age group. It is believed that vertigo occurs as a result of pressure changes in the middle ear and labyrinthitis [16]. The rate of otitis media in the present study was 3\%. Infrequently, an association can be observed in childhood between vertigo and sinusitis. In these patients, vertigo can be accompanied by headache, 
loss of balance, and nausea and vomiting [17]. In our study, six (3\%) patients were found to have sinusitis, and mastoiditis was the underlying etiology in two (1\%) patients.

Vertigo is often a subjective finding and it can be accompanied by psychiatric disorders, such as anxiety disorders, depression, and behavioral disorders [18]. In a study involving only children aged younger than 16 years, Manrique Lipa et al. [19] detected psychogenic vertigo in $10 \%$ of children with vertigo. In a study including 37 pediatric patients with vertigo, Gruber et al. [3] reported psychogenic vertigo in eight $(22 \%)$ patients. In our study, $13(7 \%)$ patients were diagnosed with psychogenic vertigo, mostly originating from anxiety and behavioral disorders.

Approximately $10 \%$ of cases of vertigo in the 56-year-old age group are associated with visual disturbances. Usually vertigo recovers when the visual disturbances are corrected. Visual disturbances can be related to refractive errors (myopia, hypermetropia or astigmatism) or ocular vergence anomalies. Vertigo perception involves the sense of spinning or rolling, can last shortly but is recurrent and often associated with fatigue [20]. The rate of vertigo associated with refractive error was $6 \%$ in the present study.

$\mathrm{S}$ uch peripheral vestibular disorders as benign paroxysmal positional vertigo (BPPV), vestibular neuritis, and Ménière's disease are uncommon in adults than in children [21]. The rate of BPPV was found to be $8 \%$ in the present study, whereas no patient had vestibular neuritis and Ménière's disease. Likewise, a history of trauma and vertigo after an operation that is considered in the differential diagnosis of vertigo was detected only in one patient. In this study, two patients with vertigo of childhood were found to have accompanying subclavian steal syndrome, which is an uncommonly reported condition, and one patient was found to have multiple sclerosis.

Autoimmune thyroiditis is the most common cause of goiter and acquired hypothyroidism among children living in iodine-sufficient areas. Patients may present with such symptoms as weakness, dry skin, constipation along with extrathyroidal symptoms, including alopecia, vitiligo, atopy, and depression [22]. In the present study, autoimmune thyroiditis was determined as the likely cause of vertigo in seven $(5 \%)$ patients. Vitamin B12 deficiency often results in hematological and neurological problems in children. Aside from anemia, infants often present with the symptoms related to the brain development and a delay in overall growth and development including hypotonia, poor feeding, glossitis, lethargy, tremor, irritability, and coma. On the other hand, older children might have peripheral neuropathy, symmetrical paresthesia, ataxia, gait disorders and even psychiatric disorders involving depression and psychosis [23]. Iron deficiency anemia in children may cause symptoms such as palpitation, fatigue, exercise intolerance, headache, irritability, and sensory disorders [24]. In our study, seven (4\%) patients had autoimmune thyroiditis, nine (5\%) had vitamin B12 deficiency, and three (2\%) patients had iron deficiency anemia.

While considering whether to perform neuroimaging studies and neurophysiological laboratory tests in patients with vertigo of childhood, potential effects of radiation must be taken into consideration along with the fact that the child may show limited cooperation in neuroimaging studies, vestibular tests and evoked potential tests that require cooperation for optimal results [3]. For example, CT has a very limited place in evaluation of vertigo in children. However, the use of MRI as a neuroimaging study has become a more common practice owing to lack of radiation exposure and its superiority in delineating posterior fossa and inner ear structures. Neuroimaging studies still contribute little to the diagnosis in patients presenting with vertigo alone. Raucci et al. [7] performed neuroimaging studies in $20.8 \%$ of 616 children who presented to the pediatric emergency room with the complaint of vertigo and detected serious neurological problems only in $2.5 \%$. Also, they noted significant neurological symptoms accompanied vertigo in all of these latter patients.

Similarly, a retrospective study of 87 pediatric patients presenting with vertigo reported new findings on neuroimaging studies in 23 patients, however, 19 out of 23 patients had neurological deficits in addition to vertigo [25]. The authors particularly emphasized that neuroimaging studies do not contribute significantly to elucidating the etiology of vertigo. In a study from Turkey, Erdoğan et al. [1] performed MR imaging of the brain in 13 out of 30 pediatric patients with vertigo and reported no pathological findings in any of these patients. Neuroimaging studies showed 
normal findings in $144(85 \%)$ out of 171 patients, whereas $17(10 \%)$ were found to have nonspecific findings (arachnoid-pineal gland cyst, corpus callosum agenesis, choroid plexus papilloma, pituitary microadenoma, arachnoid granulation), four (2\%) had sinusitis, two (1\%) had mastoiditis and subclavian steal syndrome, one patient had venous angioma and another patient had demyelinating plaques. Consistent with the literature, these results suggest that neuroimaging studies have a very limited role in the assessment of children with vertigo.

\section{CONCLUSION}

This study shows that orthostotic hypotension and BPPV are the most common causes of vertigo. Autoimmune thyroiditis, vitamin B12 deficiency, iron deficiency anemia weredetected in approximately onetenth of patients, and epilepsy was diagnosed in 7\% of the patients. This shows that as in all diseases, it is important to evaluate patients systemically in vertigo. It should be kept in mind that vertigo can be an epileptic symptom. As in other studies, it was seen that neuroimaging studies did not contribute much to vertigo.

\section{Conflict of interest}

The authors disclosed no conflict of interest during the preparation or publication of this manuscript.

\section{Financing}

The authors disclosed that they did not receive any grant from funding agencies in the public, commercial, or not-for-profit sectors during conduction or writing of this study.

\section{REFERENCES}

1. Erdoğan E, Güzel Nur B, Olgaç Dündar N. [Vertigo in childhood: evaluation of clinical and laboratory findings]. Türkiye Klinikleri J Med Sci 2012;32:1601-6. [Article in Turkish]

2. Gioacchini FM, Alicandri-Ciufelli M,Kaleci S, Magliulo G, Re M. Prevalence and diagnosis of vestibular disorders in children: a review. Int J Pediatr Otorhinolaryngol 2014;78:71824.

3. Gruber M, Cohen-Kerem R, Kaminer M, Shupak A. Vertigo in children andaAdolescents: characteristics and outcome. Sci World J 2012;2012:109624.

4. Erbek SH, Erbek SS, Yilmaz I, Topal O, Ozgirgin N, Ozluoğlu LN, et al. Vertigo in childhood: a clinical experience. Int J Pediatr Otorhinolaryngol 2006;70:1547-54.

5. Jahn K, Langhagen T, Heinen F. Vertigo and dizziness in children. Curr Opin Neurol 2015;28:78-82.

6. Devaraja, K. Vertigo in children; a narrative review of the various causes and their management. Int $\mathrm{J}$ Pediatr Otorhinolaryngol 2018;111:32-8.

7. Raucci U, Vanacore N, Paolino MC, Silenzi R, Mariani R, Urbano A, et al. Vertigo/dizziness in pediatric emergency department: five years' experience. Cephalalgia 2016;36:593-8. 8. McCaslin DL, Jacobson GP, Gruenwald JM. The predominant forms of vertigo in children and their associated findings on balance function testing. Otolaryngol Clin North Am 2011;44:291-307.

9. Krams B, Echenne B, Leydet J, Rivier F, Roubertie A. Benign paroxysmal vertigo of childhood: long-term outcome. Cephalalgia 2011;31:439-43.

10. Wiener-Vacher SR. Vestibular disorders in children. Int J Audiol 2008;47:578-83.

11. Ravid S, Bienkowski R, Eviatar L. A simplified diagnostic approach to dizziness in children. Pediatr Neurol 2003;29:31720.

12. Niemensivu R, Kentala E, Wiener-Vacher S, Pyykko I. Evaluation of vertiginous children. Eur Arch Otorhinolaryngol 2007;264:1129-35.

13. Furman JM, Marcus DA, Balaban CD. Migrainous vertigo: development of a pathogenetic model and structured diagnostic interview. Curr Opin Neurol 2003;16:5-13.

14. Saltürk Z, Yıldırım G, Sünnetçi G, Uyar Y, Atar Y, Kumral TL, et al. Evaluation of vertigo in pediatric age group. Eur Arch Med Res 2014;30:57-62.

15. Kluge M, Beyenburg S, Fernandez G, Elger CE. Epileptic vertigo: evidence for vestibular representation in human frontal cortex. Neurology 2000;55:1906-8.

16. Riina N, Ilmari P, Kentala E. Vertigo and imbalance in children: a retrospective study in a Helsinki University otorhinolaryngology clinic. Arch Otolaryngol Head Neck Surg 2005;131:996-1000.

17. American Academy of Pediatrics. Subcommittee on Management of S, Committee on Quality I. Clinical practice guideline: management of sinusitis. Pediatrics 2001;108:798-808. 18. Emiroğlu FN, Kurul S, Akay A, Miral S, Dirik E. Assessment of child neurology outpatients with headache, dizziness, and fainting. J Child Neurol 2004;19:332-6.

19. Manrique Lipa RD, Soto Varela A, Santos Pérez S, Manrique Lipa RK, Lorenzo Lorenzo AI, Labella Caballero T. Alterations of balance in patients under 16 years of age distributed by age groups. Acta Otorrinolaringol Esp 2008;59:455-62.

20. Bucci MP, Kapoula Z, Yang Q, Wiener-Vacher S, BremondGignac D. Abnormality of vergence latency in children with vertigo. J Neurol 2004;251:204-13.

21. Jahn K, Langhagen T, Schroeder AS, Heinen F. Vertigo and dizziness in childhood - update on diagnosis and treatment. Neuropediatrics 2011;42:129-34. 
22. Dilek E, İşçan B, Ekuklu G, Tütüncüler F. [Retrospective evaluation of the cases diagnosed as Hashimoto's thyroiditis]. Çocuk Dergisi 2011;11:73-7. [Article in Turkish]

23. Molloy AM, Kirke PN, Brody LC, Scott JM, Mills JL. Effects of folate and vitamin B12 deficiencies during pregnancy on fetal, infant, and child development. Food Nutr Bull 2008;29(2 Suppl):S101-11.
24. World Health Organization/UNICEF/UNU. Iron deficiency anemia: assessment, prevention, and control. A guide for programme managers. Geneva, Switzerland: World Health Organization; 2001.

25. Niemensivu R, Pyykko I, Valanne L, Kentala E. Value of imaging studies in vertiginous children. Int $\mathrm{J}$ Pediatr Otorhinolaryngol 2006;70:1639-44. 